\title{
Heterodyne spectroscopy with superconducting single-photon detector
}

\author{
Yu.V. Lobanov ${ }^{1,2, *}$, M.L. Shcherbatenko ${ }^{1,2}$, A.V. Semenov ${ }^{1,2}$, V.V. Kovalyuk ${ }^{1}$, \\ A.A. Korneev ${ }^{1,2}$, and G.N. Goltsman ${ }^{2,3}$ \\ ${ }^{1}$ Moscow Institute of Physics and Technology, 147000 Dolgoprudny, Russia \\ ${ }^{2}$ Moscow State Pedagogical University, 119991 Moscow, Russia \\ ${ }^{3}$ National Research University Higher School of Economics, 101000 Moscow, Russia
}

\begin{abstract}
We demonstrate successful operation of a Superconducting Single Photon Detector (SSPD) as the core element in a heterodyne receiver. Irradiating the SSPD by both a local oscillator power and signal power simultaneously, we observed beat signal at the intermediate frequency of a few MHz. Gain bandwidth was found to coincide with the detector single pulse width, where the latter depends on the detector kinetic inductance, determined by the superconducting nanowire length.
\end{abstract}

Heterodyne detection is one of the most powerful spectroscopic tools. Although it originated from the radio-frequency band, its applicability in optics has been suggested and demonstrated [1-2]. With invention of the laser, use of the heterodyne technique in optics has been increased drastically, including various applications in astrophysics, spectroscopy, lidar development, communication. With aim to improve performance of the receivers, use of photon-counting detectors has been proposed [3], and APDs array has been utilized for the lidar experiment [4]. Here we demonstrate successful operation of a Superconducting Single Photon Detector (SSPD) [5,6] as the core element for heterodyne spectroscopy.

We used two independent fibre-coupled DBF-lasers operating at wavelength near $1550 \mathrm{~nm}$ as the local oscillator (LO) and signal source. The wavelength of each laser was precisely set by adjusting its operating temperature through a computer-controlled bias unit. The output power from each laser was adjusted by optical attenuators and the polarization was adjusted by the polarization controllers. Then waveforms from LO and signal were combined by the beamsplitter and fed to the SSPD operated in liquid helium cryostat at $1.7 \mathrm{~K}$ temperature. The photon detection events - several nanoseconds-long electrical voltage pulses - were amplified by two room temperature amplifiers with $1 \mathrm{GHz}$ band and total gain of about $50 \mathrm{~dB}$ and then fed to either digital oscilloscope Rohde \& Schwartz RTO 1022 or Spectrum Analyzer R\&S FSV 13. We stored sub-millisecond train of pulses in the oscilloscope memory and then performed their statistical analysis.

When both waveforms are combined the power of the resulting field experiences beating at an intermediate frequency (IF) which is the difference between LO and signal frequencies. Consequently, the probability of detection event exhibits the same beating at IF. Fig.1 (a) demonstrates a zoomed-in view on the train of pulses recorded with the SSPD. Given the

* Corresponding author: akorneev@rplab.ru 
recorded set of detection events one can then digitally perform Fourier-transform analysis and find IF. Fig. 1 (b) presents the result of such analysis, clearly showing IF signal at $\sim 11 \mathrm{MHz}$ fitted by the Cauchy-Lorentzian distribution.

(a)
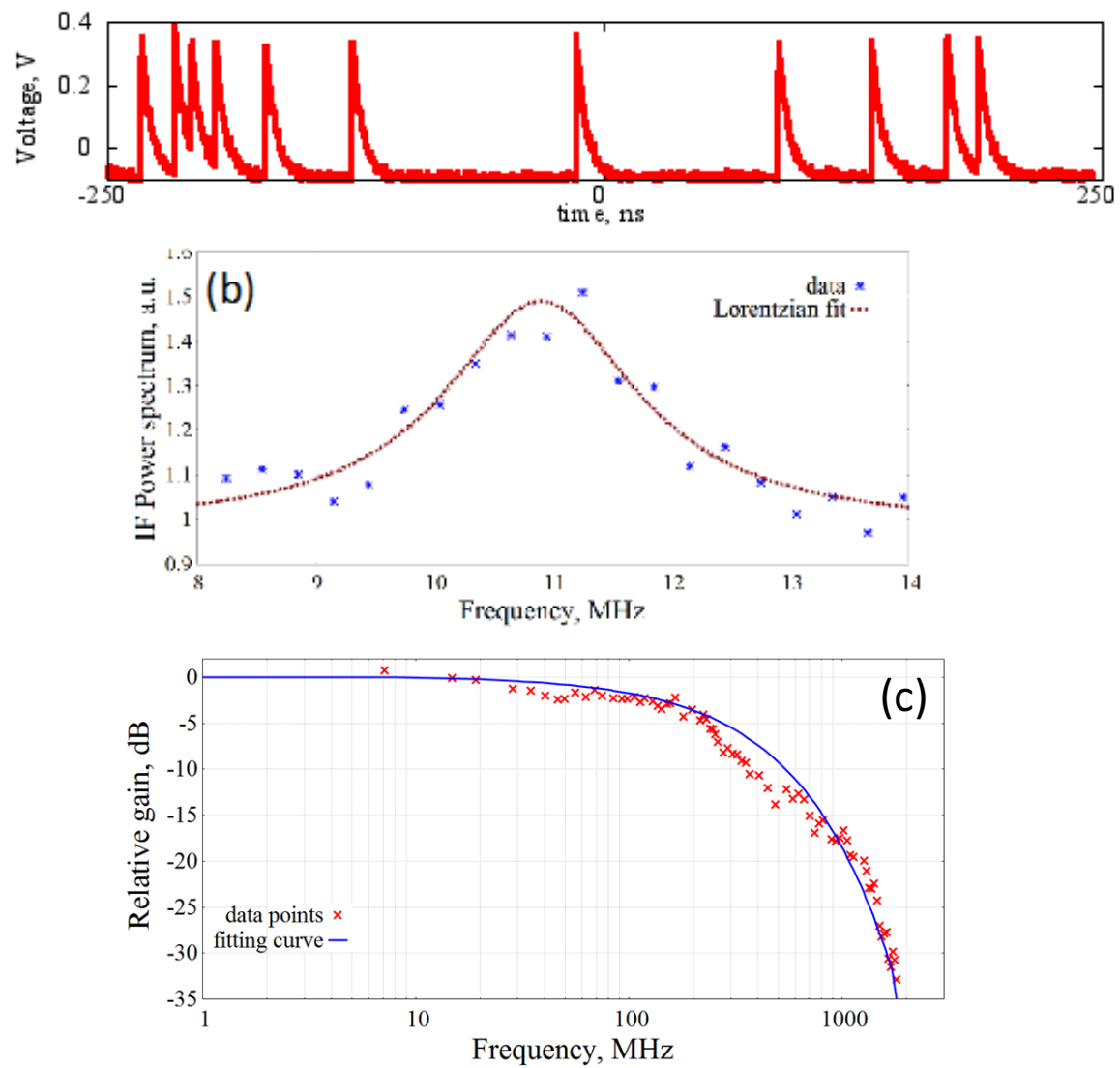

Fig. 1. (a) Trace of photon detection voltage pulses distributed with the beating frequency. (b) Result of Fourier analysis of detection events distribution in time presenting maximum at the intermediate frequency. (c) Gain bandwidth of the SSPD operated in heterodyne detection regime.

One of the important characteristics of a mixer is the mixer gain bandwidth. With the analog spectrum analyzer we measured the output power at the IF. By changing the local oscillator wavelength, we were able to determine the gain bandwidth of our system, as shown in Fig. 1 (c). The measured gain bandwidth was as large as $140 \mathrm{MHz}$.

This work was supported by Russian Science Foundation project 16-19-10633.

\section{References}

1. A.T. Forrester et al., Phys. Rev. 72, 728 (1947)

2. A.T. Forrester, R.A. Gudioindsen, P.O. Johnson, Phys. Rev. 99, 1691 (1955)

3. D.E. Sawyer, Heterodyne operation of avalanche photodiodes (NASA, USA, 1967)

4. J.X. Luu, L.A. Jiang, Appl. Opt. 45, 3798 (2006)

5. G. Gol'tsman et al., Appl. Phys. Lett. 79, 705 (2001)

6. C.M. Natarajan et al., Supercond. Sci. Technol. 25, 063001 (2012) 